\title{
Directing Min protein patterns with advective bulk flow
}

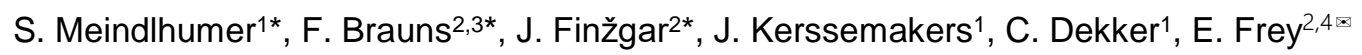

${ }^{1}$ Department of Bionanoscience, Kavli Institute of Nanoscience Delft, Delft University of Technology, Delft, the Netherlands

${ }^{2}$ Arnold Sommerfeld Center for Theoretical Physics and Center for NanoScience, Department of Physics, Ludwig-Maximilians-Universität München, Munich, Germany

${ }^{3}$ Present address: Kavli Institute for Theoretical Physics, University of California Santa Barbara, Santa Barbara, CA 93106, USA

${ }^{4}$ Max Planck School Matter to Life, Hofgartenstraße 8, 80539 Munich, Germany

* These authors contributed equally to this paper

ॠFor comments and questions please contact frey@Imu.de

\begin{abstract}
We theoretically predict and experimentally show that the propagation direction of in vitro Min protein patterns can be controlled by a hydrodynamic flow of the bulk solution. We find downstream propagation of Min wave patterns relative to the bulk flow direction for low MinE:MinD concentration ratios, but upstream propagation for large MinE:MinD ratios, with multistability of both propagation directions in between. A theoretical model for the Min system reveals the mechanism underlying the upstream propagation and links it to the fast conformational switching of MinE in the bulk. For high MinE:MinD ratios, upstream propagation can be reproduced by a reduced model in which increased MinD bulk concentrations on the upstream side promote protein attachment and hence, propagation in that direction. For low MinE:D ratios, downstream propagation is described by the minimal model, as additionally confirmed by experiments with a non-switching MinE mutant. No advection takes place on the membrane surface where the protein patterns form, but advective bulk flow shifts the proteinconcentration profiles in the bulk relative to the membrane-bound pattern. From a broader perspective, differential flows in a bulk volume relative to a surface are a relevant general feature in bulk-surface coupled systems. Our study shows how such a differential flow can control surface-pattern propagation and demonstrates how the global pattern's response may depend on specific molecular features of the reaction kinetics.
\end{abstract}


bioRxiv preprint doi: https://doi.org/10.1101/2021.12.23.474007; this version posted December 23, 2021. The copyright holder for this preprint (which was not certified by peer review) is the author/funder, who has granted bioRxiv a license to display the preprint in perpetuity. It is made available under aCC-BY-NC-ND 4.0 International license.

\section{Introduction}

Pattern formation is a phenomenon observed in widely different contexts from physics to biology. In cell biology and embryology, it has been studied across species in intracellular ${ }^{1-3}$ as well as multicellular systems ${ }^{4-6}$. The term broadly refers to the self-organization of molecules based on physicochemical principles, realized by the interplay of complex reaction networks, transport mechanisms, and guiding cues. ${ }^{1,2,4,7-10}$ Intracellular pattern formation is known to play important roles in the positioning of protein assemblies, particularly during cell division. ${ }^{1-3,7,8,11-14}$

Here, we study how advective flow affects protein-based pattern formation. Fluid flow was found to be essential for asymmetry and pattern formation in several organisms. ${ }^{15}$ A prominent example is the establishment of the posterior-anterior axis within the monocellular $C$. elegans zygote, in which actomyosin cortical flows were found to transport regulatory PAR proteins ${ }^{16-18}$ and additionally, to be associated with cytosolic streaming ${ }^{19}$. To investigate the effect of advective flow on intracellular pattern formation in a controlled setting, we here focus on a system that allows for in vitro reconstitution and controlled manipulation of system parameters.

The Min protein system from E. coli bacteria is the best studied model system for intracellular pattern formation. While rich in complexity with all its known and possible interactions within a cell and its biological role prior to Z-ring formation, ${ }^{12,20}$ it is at the same time intriguingly simple as its core patternforming mechanism essentially comes down to the interaction of only two proteins, MinD and MinE. The interaction of these proteins is widely considered the textbook example for a mass-conserving reaction diffusion system and has become the subject of numerous theoretical and experimental studies. ${ }^{21-30}$ In vitro reconstitution is well-established and relies on imaging of fluorescently labelled purified Min protein on supported lipid bilayers. ${ }^{3,24}$

MinD is an ATPase, which binds to a lipid membrane upon binding ATP in the bulk. Once bound, MinDATP recruits more of its own kind, leading to a positive feedback loop with an enhanced binding of MinDATP to the membrane in its vicinity. This process is constantly counteracted by MinE, an ATPaseactivating protein that also binds to the membrane upon getting recruited by membrane-bound MinDATP. Membrane-bound MinE triggers MinD to detach from the membrane, whereby MinD hydrolyzes its ATP in the process. Back in the bulk, MinD exchanges ADP for ATP and starts the cycle anew. 1,7,12 This simplified description (see Fig. 1B) is complemented and modified by countless details within the process, such as multimerization, ${ }^{28}$ the local MinE:MinD stoichiometry, ${ }^{29,31}$ the formation of a depletion zone, ${ }^{31}$ bulk-surface coupling, ${ }^{22}$ and (particularly notable for our study) the so-called MinE switch ${ }^{26}$. The latter describes the ability of MinE to temporarily adopt a latent, non-reactive state upon membrane detachment. Non-switching mutants of MinE that cannot access this latent state were found to still be capable of pattern formation, albeit only within an extremely reduced concentration range. The presence of the MinE switch thus increases the robustness of the Min system towards concentration fluctuations. ${ }^{26}$ Numerous strategies have been employed to study, manipulate, and take advantage of the properties of Min patterns. ${ }^{32}$ Examples include changing the membrane or buffer composition ${ }^{27}$, crafting surface topology ${ }^{33}$, microfabrication of sample chambers ${ }^{34}$, variation of sample chamber geometry ${ }^{22,30}$, 
bioRxiv preprint doi: https://doi.org/10.1101/2021.12.23.474007; this version posted December 23, 2021. The copyright holder for this preprint (which was not certified by peer review) is the author/funder, who has granted bioRxiv a license to display the preprint in perpetuity. It is made available under aCC-BY-NC-ND 4.0 International license.

exploration of cargo molecule transport ${ }^{35}$, integration with photoswitchable compounds ${ }^{36}$, liposome encapsulation ${ }^{37}$, and de novo synthesis within liposomes ${ }^{38}$.

In an in vitro study by Vecchiarelli et al, ${ }^{27}$ external hydrodynamic flow was shown to influence Min protein patterns. More specifically, Min-protein patterns were observed to propagate upstream under fast bulk flow, meaning that they formed waves that were traveling against the direction of the hydrodynamic flow. The authors hypothesized that the cause of this upstream propagation was advective transport of reactive MinE in the vicinity of the membrane - a hypothesis which, to the best of our knowledge, has not been tested to date. To our understanding, an important implication of this finding is that bulk flow can be used to probe molecular mechanisms - motivating us to systematically study the effect of bulk flow on pattern formation in the Min protein system.

In the investigations presented here, we combine numerical simulations of theoretical models and in vitro experimental analysis to study the influence of bulk fluid flow on Min protein patterns. We show that bulk flow promotes the emergence of wave fronts traveling in the flow direction, much like for water surface waves. Interestingly, not only the speed but also their direction of propagation is found to depend critically on the concentration ratio between MinE and MinD, henceforth referred to as the E:D ratio.

We predict and experimentally show that, surprisingly, wavefronts tend to propagate upstream (against the direction of bulk flow) for high E:D ratio, but downstream (along the direction of bulk flow) for low E:D ratio. While these results were obtained using our full Min model (including the MinE switch ${ }^{26}$, see Fig. 1B), we additionally developed simplified models for specifically the high and low E:D regimes, in order to gain a better understanding of which mechanisms dominate in either of these cases. These simplified models take the different effects of the MinE switch into account and were found to reproduce the results of the full model when operating in the respective regime. We refer to these simplified models as the reduced switch model for high E:D ratio (Fig. 1E) and the skeleton model for low E:D ratio (Fig. $1 F)$. To test the skeleton model, we also performed experiments with a non-switching mutant MinE$\mathrm{L} 3 \mathrm{I} 24 \mathrm{~N}^{26}$ and found that it only showed downstream propagation, as predicted by the model.

Additionally, our simulations and experiments show that not only the E:D ratio, but also the magnitude of the bulk flow rate determines the pattern's properties. We found that lower rates promote downstream, while higher rates did promote upstream propagation. Particularly for intermediate E:D ratios, we predicted phenomena such as hysteresis and multistability, leading to either downstream or upstream propagation depending on initial settings. Experimental exploration of this regime revealed that the outcome is indeed far from binary, as different positions within the same sample were found to respond differently to the same external perturbation (flow). We developed image analysis methods that allow us to statistically evaluate a given pattern's response to flow, with results in overall qualitative agreement with our simulations. Combined, our findings provide further insight into how local Min protein interactions lead to large-scale pattern formation. 
bioRxiv preprint doi: https://doi.org/10.1101/2021.12.23.474007; this version posted December 23, 2021. The copyright holder for this preprint (which was not certified by peer review) is the author/funder, who has granted bioRxiv a license to display the preprint in perpetuity. It is made available under aCC-BY-NC-ND 4.0 International license.

\section{Results}

\section{MinE-to-MinD concentration ratio determines propagation direction}

Our primary goal is to study the qualitative response of Min patterns to external fluid flow. For simplicity, we consider uniform laminar flow and use a previously established, parsimonious model ${ }^{26}$ for the Min reaction kinetics as depicted in Fig. 1B. Since a previous experimental study hypothesized that switching of MinE between reactive and latent states in the bulk is responsible for the upstream propagation of Min patterns, ${ }^{27}$ we explicitly include this conformational switching of MinE in our model, which we accordingly refer to as the full model. ${ }^{26}$

We performed finite element simulations in a rectangular area representing the lipid bilayer membrane and the bulk solution above it, choosing periodic boundary conditions in the lateral directions to reduce finite-size effects. The dimension orthogonal to the membrane was integrated out (and explicitly accounting for this dimension does not change the qualitative findings, see SI). We performed simulations for different $E: D$ ratios because previous studies did show that the concentrations of MinD and MinE, and in particular their E:D ratio, are essential control parameters for Min protein pattern formation. ${ }^{31}$

As illustrated in Fig. 1C and Fig. 1D, our simulations show that uniform flow has two main effects: (i) Wave fronts align perpendicular to flow direction and (ii) the wave propagation direction aligns upstream (against the flow) or downstream (with the flow), depending on the E:D ratio. While upstream propagation occurs for high E:D ratios (movie 1), downstream propagation is found for low E:D ratios (movie 2). Notably, we predict downstream propagation in regimes that would not allow for pattern formation in the absence of flow. We refer to this phenomenon as a flow-driven instability. ${ }^{39,40}$ (Please see SI Sec. 1.7.) Moreover, we observed that downstream propagating patterns slowly increase in wavelength, in a process reminiscent of coarsening dynamics in phase-separating systems. Eventually, only a single propagating soliton-like pulse remained in the simulation domain, as shown in Fig. 1D.

To test our theoretical predictions, we performed experiments with purified Min proteins in flow channels that were coated with a lipid bilayer that mimics the cell membrane. Min proteins readily formed patterns on the supported lipid bilayer composed of a mixture of DOPC and DOPG at a 2:1 ratio matching the negative charge density of the $\mathrm{E}$. coli membrane. ${ }^{27}$ To reach high flow rates while minimizing the required amount of protein, a closed-circle system was used for all experiments with wildtype MinE. As schematically shown in Fig. S6, the protein solution from the flow channel's outlet was pumped back to its inlet via a closed tubing system. The concentration loss due to sticking was later estimated from protein gels, allowing to calculate corrected E:D ratios, given in brackets. As the mutant MinE-L3I24N exhibits pattern formation only for a very constricted range of concentrations ${ }^{26}$, closed-circle experiments were found less suitable due to protein sticking, and an open system was used instead.

As we found that mere visual inspection was too unreliable to determine the patterns' response to flow, we developed automatized tools that allowed us to quantify the propagation speed and direction of wave crests. ${ }^{41}$ We identified individual crest points, determined how they moved frame-by-frame and 
bioRxiv preprint doi: https://doi.org/10.1101/2021.12.23.474007; this version posted December 23, 2021. The copyright holder for this preprint (which was not certified by peer review) is the author/funder, who has granted bioRxiv a license to display the preprint in perpetuity. It is made available under aCC-BY-NC-ND 4.0 International license.

calculated their translocation vector components (see Materials \& Methods and SI). Fig. 2 shows exemplary images along with the results from the crest velocity analysis (collected from up to three comparable imaging regions within one flow cell) visualized as 2D histograms. We experimentally found that an applied advective flow had multiple effects on the Min patterns. We observed a clear decrease in the occurrence of spiral patterns, as patterns tended to transition into traveling waves with wavefronts aligned orthogonal to the flow direction. Importantly, the traveling waves that formed during an applied flow, exhibited upstream propagation for high E:D ratio ( $E: D=10$, Fig. $2 A$ and movie 3 ), and downstream propagation for low $\mathrm{E}: \mathrm{D}$ ratio ( $\mathrm{E}: \mathrm{D}=2$, Fig. $2 \mathrm{~B}$ and movie 4). Notably, in a control experiment we reversed the flow rate and found that the pattern's propagation direction also reversed several minutes (see Fig. S10 and movie 3).

All these experimental observations are in good qualitative agreement with the simulation results. Most importantly, we predicted from our simulations that the relative concentration of MinE with respect to MinD would lead to different outcomes with respect to the patterns' directionality relative to the external flow. There are, however, also notable differences, both qualitative and quantitative. An example of the former is that downstream waves are experimentally observed for E:D ratios that also exhibit pattern formation without any applied flow. This finding stands in contrast to our simulations, where downstream propagation only appears in regimes which would not allow for pattern formation in the absence of flow. Further, we do not observe the predicted coarsening (i.e., a strong increase of wavelength) for downstream propagating waves in our experiments.

A clear quantitative difference is the value for the $E: D$ ratio above which upstream propagation can be observed. In experiments, we had to go to much higher E:D ratios $(>2)$ than in the simulations to get upstream propagation, where we observed it starting from E:D ratios of 0.1 (Fig. 3).

\section{Model reductions and mechanistic explanation of upstream propagation}

Reducing a model to the key features dominating within a given parameter regime is a strategy that can provide important insights into the mechanisms underlying the entire process described by the model. To gain intuition about the origin of the E:D dependence of a pattern's response to bulk flow, we studied two reduced models that reproduce the results of the full model in the limits of high and low E:D ratio, respectively.

In the limit of large MinE concentration and fast switching of MinE between the reactive and latent conformations, the MinE switching dynamics can be eliminated using a quasi-steady state approximation (see SI). The resulting reduced switch model, visualized by the network cartoon in Fig. $1 \mathrm{~F}$, exclusively exhibits upstream propagation in response to flow. This is consistent with the numerical simulations of the full model in the regime of large E:D ratio (see Fig. 3). In the limit where the reduced switch model is valid, bulk concentration gradients of MinE are negligible such that MinE bulk concentrations no longer appear explicitly as dynamic variables. This in turn implies that advective transport of MinE has no effect on the dynamics. As an additional test, we performed simulations of the full model where MinE is not advected by the flow. Consistent with our expectation from the theoretical 
bioRxiv preprint doi: https://doi.org/10.1101/2021.12.23.474007; this version posted December 23, 2021. The copyright holder for this preprint (which was not certified by peer review) is the author/funder, who has granted bioRxiv a license to display the preprint in perpetuity. It is made available under aCC-BY-NC-ND 4.0 International license.

analysis, we found upstream propagation. Taking the above results together, we conclude that contrary to an earlier hypothesis ${ }^{27}$ - upstream propagation of Min-protein patterns is not due to downstream transport of (reactive) MinE.

This naturally raises the question of what the actual cause of upstream propagation is. To understand this, we consider an incipient accumulation zone of MinD on the membrane. Recruitment of MinD to the membrane acts as a sink, such that the neighboring bulk region becomes depleted of MinD (see Fig. 1A). This depletion zone is replenished by diffusion, and at the same time transported downstream by the bulk flow. This downstream transport accelerates the replenishment on the upstream side of the accumulation zone and thus, allows faster recruitment of MinD there. Vice versa, recruitment on the downstream side is reduced. As a net effect, one obtains an upstream movement of the accumulation zone (while the individual proteins do not move laterally). This differential flow-induced propagation has been previously studied for two-component mass-conserving reaction diffusion systems. ${ }^{42}$ There, it was shown that flow drives the upstream propagation of patterns that are stationary in the absence of flow.

Let us now turn to the regime of low E:D concentration ratio in which we observe downstream propagation of the membrane-bound protein waves. Previous studies showed that conformational switching of MinE can be neglected in this regime. ${ }^{22}$ This is because the majority of MinE (within the penetration depth of bulk gradients orthogonal to the membrane) is in the reactive form and rapidly cycles between membrane and bulk. Indeed, in simulations of a reduced skeleton model that does not include MinE conformational switching, we exclusively found downstream propagating waves, consistent with the notion that the model captures the relevant dynamics in the low E:D regime. To experimentally test this rationale, we replaced MinE with a non-switching mutant MinE-L3I24N ${ }^{26}$ and indeed found downstream propagating waves only, as shown in Fig. $2 \mathrm{C}$ and movie 5. Notably, we find that pattern formation with this MinE mutant requires sufficiently low E:D ratios in agreement with previous experiments and theory. ${ }^{26}$

Next, we tried to decipher the mechanism of downstream propagation. Here, the situation is much more convoluted than in the regime of high $\mathrm{E}: \mathrm{D}$, because here bulk gradients of both MinD and MinE are significant. While MinD recruits itself to the membrane, MinE recruited by MinD drives MinD detachment by catalyzing MinD hydrolysis. Intuitively, one might think that the above reasoning for flow-induced upstream propagation might be applied to explain downstream propagation based on MinE advection and the MinE-driven MinD detachment. The reasoning would be that MinE is replenished faster and therefore, recruited faster on the upstream side of the MinD-accumulation zone. This would result in faster MinD detachment there, compared to the downstream side, resulting in a downstream propagation of the MinD-accumulation zone. To test this intuition, we performed simulations in which MinE was not advected by the flow. Strikingly, we still observe downstream propagating waves (see Fig. S4). This indicates that an intricate interplay of advective MinD transport and diffusive MinE transport is responsible for downstream propagation, whereas MinE advection is not crucial. Disentangling this interplay remains an open challenge for future research. 
bioRxiv preprint doi: https://doi.org/10.1101/2021.12.23.474007; this version posted December 23, 2021. The copyright holder for this preprint (which was not certified by peer review) is the author/funder, who has granted bioRxiv a license to display the preprint in perpetuity. It is made available under aCC-BY-NC-ND 4.0 International license.

\section{Hysteresis and transition to upstream propagation by increasing flow rate}

Next, we next turned to two closely connected questions: How does the propagation direction transition from upstream to downstream (movie 6) at intermediate E:D ratios? How does the flow speed impact the dynamics?

To address these points, we mapped out a two-dimensional phase diagram employing finite-element simulations using the flow speed and the E:D ratio as control parameters. The resulting phase diagram is shown in Fig. 3A.

A striking feature of this phase diagram is that increasing the flow rate can drive a reversal from downstream propagation to upstream propagation for intermediate E:D ratios. Importantly, we find that this transition shows hysteresis, meaning that the point on which the transition occurs depends on whether one increases or decreases the flow rate or the E:D ratio. Consequently, there is a regime in which propagation in either direction is possible as the propagation direction sensitively depends on the initial conditions and history. For the downstream to upstream transition, we also observed a correlation with the pattern wavelength (see Fig. S2). Downstream propagating patterns were found to slowly coarsen in our simulations (increasing their wavelength). We observed that the downstream-toupstream transition occurred at higher flow rates for longer wavelength patterns (see Fig. 3B).

In a linear stability analysis of the homogeneous steady state, we find two distinct instabilities in the multistable region: One at short wavelengths, corresponding to upstream propagating waves (as indicated by the imaginary part of the growth rate, see $\mathrm{SI}$ ), and another one at long wavelengths, corresponding to downstream propagating waves. We find that the onset of the first instability (at short wavelengths) precisely coincides with the transition from upstream to downstream propagating waves in simulations with adiabatically decreasing flow velocities. This suggests that upstream propagating waves emerged from this instability. In contrast, we did not find a characteristic feature in the linear stability properties (encoded in the dispersion relation) that corresponds to the transition from downstream to upstream propagation upon increasing flow rate.

We tested the predicted hysteresis and multistability of the Min patterns experimentally. Taking advantage of our closed-circle experimental setup, we could incrementally increase the flow rate and acquire protein patterns at distinct points (flow rate, E:D ratio) in the parameter space. Note however, that for practical reasons (such as long incubation/equilibration times), the experimental approach is not identical to the one followed in the simulations depicted in Fig. 3A. In the simulation, we started from a homogeneous steady state at a certain E:D ratio and directly started any given flow rate. In the experiment, we first established patterns in the absence of flow, and then incrementally went through a sequence of flow rates, with an associated waiting time (15-30 minutes) at each point.

An example image series of Min patterns at different bulk flow rates is displayed in Fig. 4A. Analysis of the crest propagation directions as dependent on the bulk flow rate is provided in Fig. 4B to 4E for different $E: D$ ratios in the intermediate regime. Both upstream and downstream propagating patterns 
bioRxiv preprint doi: https://doi.org/10.1101/2021.12.23.474007; this version posted December 23, 2021. The copyright holder for this preprint (which was not certified by peer review) is the author/funder, who has granted bioRxiv a license to display the preprint in perpetuity. It is made available under aCC-BY-NC-ND 4.0 International license.

were observed. Fig. 4F shows an overview on the peak velocities obtained for different E:D ratios and flow rates. The full crest velocity analysis of all experiments can be found in the SI.

To obtain a quick overview on a pattern's response to flow, we calculated angles from the vectoral components obtained from our wave propagation analysis, binned them in segments of $15^{\circ}$ and plotted their normalized occurrence for different flow rates, as shown Fig. 4B to 4E. This can be understood as a summation over counts found in a certain angular segment from a 2D histogram plot of $\left(v_{x}, v_{y}\right)$ such as those shown in Fig. 2. In Fig. 4G, we show overviews on results obtained from all experiments done with wildtype MinE. Defining the $-30^{\circ}$ to $+30^{\circ}$ segment as "downstream" (shaded red in Fig. 4B to 4E) and $150^{\circ}$ to $210^{\circ}$ as "upstream" (shaded blue in Fig. 4B to 4E), we show the downstream and upstream fractions as red and blue segments respectively, with the symbol size corresponding to the occurrence. At the lowest $E: D$ ratio, downstream propagation is clearly favored upon exposing a pattern to flow, while at the highest $E: D$ ratio, upstream propagation is dominant. For intermediate $E: D$ ratios, the outcome is less clear. Here, we found that the propagation direction sensitively depended on the initial condition, i.e. on the initial propagation direction in the absence of flow.

For most E:D ratios, we observed that waves tended to slow down upon increasing the flow rate (see Fig. 4F and Fig. S11). We were able to confirm that the observed slowing down is indeed induced by the flow and not merely a consequence of the experiment's duration. In a control experiment, the Min pattern in a sample channel was not exposed to flow yet observed over the same time period as a flowexperiment that was run in parallel in a separate flow channel (at an initial E:D ratio of 3 . The results of both the control and regular experiment are shown Fig. S8 and Fig. S9. Analysis clearly showed that while the control's pattern did change over time, it did not show the distinctive directional features of the pattern exposed to bulk flow. Slowing down of the wave crest was present and possibly linked to ATP depletion, yet less pronounced than for the flow-experiment.

\section{Discussion}

In our study, we performed simulations as well as in vitro experiments designed to investigate the influence of advective bulk flow on membrane-bound protein patterns. We theoretically predicted and experimentally showed that Min protein patterns respond differently to hydrodynamic flow depending on the $E: D$ ratio and flow rate. The transition from upstream propagation (high $E: D$ ) to downstream propagation (low E:D) is qualitatively captured by a parsimonious model that explicitly accounts for conformational switching of MinE. For intermediate $E: D$ ratios, our model predicts multistability of waves propagating in either direction, resulting in hysteresis. This imparts a strong dependence of the dynamics on the initial state, which we also observed in our experiments for intermediate E:D ratios (Fig. 4G). Our analysis shows that different pattern-forming mechanisms operate in low and high E:D conditions, with the role of MinE bulk gradients being the key difference between these mechanisms. For large $E: D$ ratios, we found MinE bulk gradients along the membrane to be negligible. In this way, we were able to reduce the full model to a simplified, effective model for MinD dynamics that allowed us to understand the mechanism of upstream propagation. In particular, we have shown that upstream propagation is not caused by MinE advection, but by a difference in MinD bulk gradients which promote attachment on the 
bioRxiv preprint doi: https://doi.org/10.1101/2021.12.23.474007; this version posted December 23, 2021. The copyright holder for this preprint (which was not certified by peer review) is the author/funder, who has granted bioRxiv a license to display the preprint in perpetuity. It is made available under aCC-BY-NC-ND 4.0 International license.

upstream side. At low E:D ratios, MinE conformational switching can be neglected, as we showed both by numerical simulations and experiments using a non-switching MinE mutant. Although much has been elucidated about the role of bulk flow on pattern formation, the mechanism underlying downstream propagation at low E:D ratios remains unclear at this point.

The key feature of our studied system is differential flow, i.e. the advection of different components (protein concentrations) with different velocities ${ }^{39}$ where bulk flow leads to advection of the proteins in solution, but not those on the membrane where the observed pattern forms. Therefore, bulk flow affects the patterns only indirectly, through the bulk-surface coupling ${ }^{22}$ caused by the attachment and detachment of proteins at the membrane surface. As a result of this indirect coupling between the protein pattern and the hydrodynamic flow, the patterns can propagate both upstream and downstream relative to the flow direction, depending sensitively on various molecular aspects of the reaction kinetics such as the attachment-detachment dynamics. The patterns' response to flow can therefore be used as a robust, qualitative observable that allows to identify regimes where different pattern-forming mechanisms operate. Bulk-surface coupling is a general feature of protein-based pattern formation. ${ }^{1}$ Differential flow will generally occur when bulk flows (e.g. cytoplasmic streaming) are present in such systems. ${ }^{43}$ On a larger scale, bulk-surface coupling is important for morphogenesis, where epithelial sheets surround fluid-filled lumens. ${ }^{44,45}$ Signaling molecules released to the lumen will be subjected to advective flows therein. By contrast, molecules that diffuse directly from cell to cell, e.g., through gap junctions, are not subject to such flows. Here, secretion and receptor-binding are the analogs to detachment and attachment in the Min system.

From a broader perspective, advective flow is a perturbation that breaks a symmetry of the system by imposing a preferred spatial direction. Pattern formation is innately connected to symmetry breaking, to the point where the terms are sometimes even used interchangeably. ${ }^{46,47}$ In the absence of spatial cues, symmetry breaking happens spontaneously due to the amplification of small random fluctuations or small heterogeneities within the system. As exemplified by Min patterns in the absence of flow or other cues, the propagation direction of waves is random and there is no predominant direction on average. Advective bulk flow breaks this symmetry, causing the wave patterns to align in a particular direction, either with or against the flow (as we showed). Thus, the bulk flow can be thought of as an analogue to an external magnetic field applied to a ferromagnetic material. Using such symmetry-breaking perturbations to probe these materials has provided valuable insights into the underlying physics. Here, we demonstrated that a related approach can be applied to a complex pattern-forming system that operates far from equilibrium.

Taking the experimentally observed responses to flow into account puts constraints on theoretical models. We tested two other Min models from the literature (developed by Bonny et al ${ }^{48}$ and Loose et $\mathrm{a}^{49}$ ) for their propensity to produce both upstream and downstream propagating patterns (see SI). We found that these models produced only downstream propagating patterns, even upon expanding them to include MinE-conformational switching. Thus, while our model predicted upstream and downstream propagation in qualitative agreement with experiments, we found that the other tested Min models did not. However, although our simulations and in vitro experiments yielded a very similar qualitative 
behavior of the influence of advective bulk flow on membrane-bound Min protein patterns, shortcomings of our own model became apparent upon making quantitative comparisons to experiments. We were unable to quantitatively determine the critical $E: D$ ratio at which the transition from downstream to upstream propagation occurs, but instead found a difference of more than an order of magnitude between the predicted and observed transition ratio. Moreover, the predicted strong increase in wavelength of downstream propagating patterns was not observed experimentally (compare Fig. 1D and Fig. S12).

To us, this suggests that additional molecular features of the protein reaction network, not yet accounted for by the current Min models, are necessary to quantitatively explain the observed phenomena. Identifying theoretical models that allow for a fully quantitative fit of the spatiotemporal dynamics of the Min system remains an open issue and active topic of research. The same holds true for the influence of advective flow on biological pattern formation, which has rarely been studied, both experimentally and theoretically. ${ }^{16,27,40,42,50-52}$ Insight into the detailed biochemical mechanisms of the MinD-MinE interactions (such as cooperative MinD self-recruitment, dimerization of MinD and MinE, MinE membrane binding, etc.) is likely needed to make progress.

To conclude, the application of hydrodynamic flow exposed the limitations of the current models and yielded additional data that can be used to constrain models in future studies. Microfluidic applications could take advantage of Min patterns where bulk flow can be used to orient membrane-bound protein patterns and adjusting the E:D ratio allows one to decide whether one wants the protein waves to go with or against the flow. Combined with the Min system's capacity for cargo transport, ${ }^{35}$ this could offer a platform for directed transport of other membrane-associated proteins. 


\section{Materials \& Methods}

Please find theoretical methods as well as extended experimental methods in the SI.

\section{Sample preparation}

Chemicals were bought from Sigma Aldrich unless specified otherwise. Flow cells were assembled using cover and microscope slides cleaned by sonication and acid Piranha, using Parafilm as a spacer, and sealed by melting the Parafilm on a hotplate. Flow channels were about $25 \mathrm{~mm}$ long, $3 \mathrm{~mm}$ wide and $200 \mu \mathrm{m}$ high, i.e. well above the threshold of tens of microns, below which coupling between upper and lower surfaces were observed. ${ }^{22}$ Exact channel heights and widths for individual experiments are given in Tab. S3. Sample channels were coated with lipid bilayers composed of DOPC:DOPG in a molar ratio 67:33 substituted with 0.01-0.02\% mol TopFluor Cardiolipin, where the latter allowed us to confirm full bilayer formation before the experiment. SUVs for lipid bilayer formation were prepared via swelling followed by stepwise extrusion with final pore size of $40 \mathrm{~nm}$. Before the experiment, chambers were filled with SUV solution, incubated for $1 \mathrm{~h}$ at $37^{\circ} \mathrm{C}$, then rinsed thoroughly with Min buffer $(150 \mathrm{mM} \mathrm{KCl}$, $25 \mathrm{mM}$ TRIS pH 7.45, $5 \mathrm{mM} \mathrm{MgCl}$ ). Next, the chamber (as well as the tubing for closed-circle experiments) were filled with Min protein solution comprising $1 \mu \mathrm{M}$ MinD and MinE at a concentration in $\mu \mathrm{M}$ equal to that of the $\mathrm{E}: \mathrm{D}$ ratio indicated (both including labelled fraction). The protein solution was supplemented with $5 \mathrm{mM}$ ATP (Thermo Fisher) as well as $5 \mathrm{mM}$ Phosphoenolpyruvic acid (Alfa Aesar) and $0.01 \mathrm{mg} / \mathrm{mL}$ pyruvate kinase for ATP regeneration. In order to study a wide range of flow rates, experiments with wildtype MinE were done in closed-circle systems (see Fig. S6). Here, the tubing was inserted into the pump, filled with protein solution and then connected to the pre-filled flow channel via two ports at its end. This allowed repeated recycling of the protein within the closed system. To check whether protein concentrations changed due to sticking to the comparably large internal surfaces of the tubing, we collected a portion of the original solution as well as the sample extracted from the closedcircle system at the end of every experiment and ran them side-by-side on a gel (see Fig. S7) to calculate an estimate for the loss, which typically was $\sim 30 \%$ for MinD and $\sim 50 \%$ for MinE. In the experiments described, the corrected ratios are given along with the initial ratios. Experiments with MinE-L3I24N were performed with an open system, with the channel's outlet connected to the pump via tubing and its inlet connected to a reservoir of protein solution, in order to minimize loss of protein due to sticking. Laminar hydrodynamic flow was created using a pressure-driven pump (Ismatec, model IPC).

\section{$\underline{\text { Image acquisition }}$}

Images were acquired using an Olympus IX-81 inverted microscope equipped with an Andor Revolution XD spinning disk system and a 20x objective (Olympus PlanN 20x / 0.4 NA). For excitation of MinDCy3 and MinE-Cy5, laser lines $561 \mathrm{~nm}$ and $640 \mathrm{~nm}$ were used. Images were acquired at multiple positions in $3 \times 3$ grids with some overlap (each single image $512 \times 512$ pixels, $594 \mathrm{~nm} /$ pixel) at $15 \mathrm{~s}$ intervals (4 frames per minute). As shown in Fig. S6, single images were stitched together to obtain larger fields of view. Up to three comparably sized regions per sample were imaged (example given in Fig. S14), obtaining total covered areas of up to $2 \mathrm{~mm}^{2}$. Regions were located in the central third of the 
channel to avoid possible turbulence close to the inlet and outlet. Following 1 hour of incubation at room temperature, images were first recorded without flow at the selected positions in order to get a reference for comparing subsequential acquisitions to. The flow rate was then set and increased incrementally as indicated. Up to 20 frames were recorded per area and flow rate. The average cross-sectional flow rate (in $\mathrm{mm} / \mathrm{s}$ ) was calculated from the flow channel's width and height as well as the pump's set flow rate (in volume/time). Images at the chosen flow rates and positions were recorded following at least $15 \mathrm{~min}$ of incubation, as we empirically found this to be about the time the pattern needed to respond to the new flow rate.

\section{$\underline{\text { Image analysis }}$}

Image cleaning, stitching as well as wave crest detection and propagation analysis were done using custom-built MatLab scripts. In brief, we identified wave crests in each frame within a stack using phase images, then compared sequential images to obtain the translation of each crestpoint frame to frame. The result of our analysis is a collective list of vectors $\left(v_{x}, v_{y}\right)$ found at positions $(x, y)$ of individual frames, collected from up to three comparable imaging regions (compare Fig. S14). These vectors were statistically analyzed with respect to their magnitude and directionality. Very low propagation velocities were cropped to eliminate the influence of static objects (protein aggregates) that would otherwise show in the results. Velocity vectors were excluded if they had a magnitude below $10 \%$ of the mean value (20\% for the highest ratio $E: D=10$, due to high background and higher occurrence of aggregates). Analysis was routinely performed on both MinE-Cy5 and MinD-Cy3 channels. In most cases, MinE-Cy5 would yield a better image quality and therefore, analysis for this channel is shown unless stated otherwise. As MinD and MinE waves are known to travel together, no qualitative difference between performing analysis for either of the fluorescence channels is to be expected. The full analysis results for all experiments performed can be found in the SI (see Fig. S15-S21).

\section{Acknowledgements}

We thank Petra Schwille at the MPI of Biochemistry Martinsried for kindly providing plasmids for overexpression of MinE-L3I24N. Further, we thank Jaco van der Torre for wet lab support, Jérémie Capoulade for support with spinning disc confocal microscopy and finally, Eli van der Sluis and Ashmiani van den Berg for purification of MinE-L3I24N. We acknowledge the BaSyC consortium for funding. J. Finžgar acknowledges the Ad Futura Scholarship (244. javni razpis) from the Public Scholarship, Development, Disability and Maintenance Fund of the Republic of Slovenia.

\section{Data and code availability}

The code and raw data produced within this study are available upon request. Please contact the corresponding author for more information. 


\section{References}

1. Halatek, J., Brauns, F. \& Frey, E. Self-organization principles of intracellular pattern formation. Phil. Trans. R. Soc. B 373, 20170107 (2018).

2. Kretschmer, S. \& Schwille, P. Pattern formation on membranes and its role in bacterial cell division. Current Opinion in Cell Biology 38, 52-59 (2016).

3. Wettmann, L. \& Kruse, K. The Min-protein oscillations in Escherichia coli : an example of self-organized cellular protein waves. Phil. Trans. R. Soc. B 373, 20170111 (2018).

4. Schweisguth, F. \& Corson, F. Self-Organization in Pattern Formation. Developmental Cell 49, 659-677 (2019).

5. Kondo, S., Watanabe, M. \& Miyazawa, S. Studies of Turing pattern formation in zebrafish skin. Phil. Trans. R. Soc. A. 379, 20200274 (2021).

6. Hatini, V. \& DiNardo, S. Divide and conquer: pattern formation in Drosophila embryonic epidermis. Trends in Genetics 17, 574-579 (2001).

7. Frey, E., Halatek, J., Kretschmer, S. \& Schwille, P. Protein Pattern Formation. in Physics of Biological Membranes (eds. Bassereau, P. \& Sens, P.) 229-260 (Springer International Publishing, 2018). doi:10.1007/978-3-030-00630-3_10.

8. Halatek, J. \& Frey, E. Rethinking pattern formation in reaction-diffusion systems. Nature Phys 14, 507-514 (2018).

9. Agudo-Canalejo, J., Illien, P. \& Golestanian, R. Cooperatively enhanced reactivity and "stabilitaxis" of dissociating oligomeric proteins. Proc Natl Acad Sci USA 117, 1189411900 (2020).

10. Wigbers, M. C., Brauns, F., Hermann, T. \& Frey, E. Pattern localization to a domain edge. Phys. Rev. E 101, 022414 (2020).

11. Thalmeier, D., Halatek, J. \& Frey, E. Geometry-induced protein pattern formation. Proc Natl Acad Sci USA 113, 548-553 (2016).

12. Ramm, B., Heermann, T. \& Schwille, P. The E. coli MinCDE system in the regulation of protein patterns and gradients. Cell. Mol. Life Sci. 76, 4245-4273 (2019).

13. Nußbaum, P. et al. An Oscillating MinD Protein Determines the Cellular Positioning of the Motility Machinery in Archaea. Current Biology S0960982220314433 (2020) doi:10.1016/j.cub.2020.09.073. 
14. Kretschmer, S., Ganzinger, K. A., Franquelim, H. G. \& Schwille, P. Synthetic cell division via membrane-transforming molecular assemblies. BMC Biol 17, 43 (2019).

15. Illukkumbura, R., Bland, T. \& Goehring, N. W. Patterning and polarization of cells by intracellular flows. Current Opinion in Cell Biology 62, 123-134 (2020).

16. Munro, E., Nance, J. \& Priess, J. R. Cortical Flows Powered by Asymmetrical Contraction Transport PAR Proteins to Establish and Maintain Anterior-Posterior Polarity in the Early C. elegans Embryo. Developmental Cell 7, 413-424 (2004).

17. Goehring, N. W. et al. Polarization of PAR Proteins by Advective Triggering of a PatternForming System. Science 334, 1137-1141 (2011).

18. Gross, P. et al. Guiding self-organized pattern formation in cell polarity establishment. Nat. Phys. 15, 293-300 (2019).

19. Niwayama, R., Shinohara, K. \& Kimura, A. Hydrodynamic property of the cytoplasm is sufficient to mediate cytoplasmic streaming in the Caenorhabiditis elegans embryo. Proceedings of the National Academy of Sciences 108, 11900-11905 (2011).

20. Bisicchia, P., Arumugam, S., Schwille, P. \& Sherratt, D. MinC, MinD, and MinE Drive Counter-oscillation of Early-Cell-Division Proteins Prior to Escherichia coli Septum Formation. mBio 4, (2013).

21. Halatek, J. \& Frey, E. Highly Canalized MinD Transfer and MinE Sequestration Explain the Origin of Robust MinCDE-Protein Dynamics. Cell Reports 1, 741-752 (2012).

22. Brauns, F. et al. Bulk-surface coupling identifies the mechanistic connection between Min-protein patterns in vivo and in vitro. Nat Commun 12, 3312 (2021).

23. Ivanov, V. \& Mizuuchi, K. Multiple modes of interconverting dynamic pattern formation by bacterial cell division proteins. Proceedings of the National Academy of Sciences 107, 8071-8078 (2010).

24. Mizuuchi, K. \& Vecchiarelli, A. G. Mechanistic insights of the Min oscillator via cell-free reconstitution and imaging. Phys. Biol. 15, 031001 (2018).

25. Loose, M., Fischer-Friedrich, E., Herold, C., Kruse, K. \& Schwille, P. Min protein patterns emerge from rapid rebinding and membrane interaction of MinE. Nat Struct Mol Biol 18, 577-583 (2011).

26. Denk, J. et al. MinE conformational switching confers robustness on self-organized Min protein patterns. Proc Natl Acad Sci USA 115, 4553-4558 (2018). 
27. Vecchiarelli, A. G., Li, M., Mizuuchi, M. \& Mizuuchi, K. Differential affinities of MinD and MinE to anionic phospholipid influence Min patterning dynamics in vitro: Flow and lipid composition effects on Min patterning. Molecular Microbiology 93, 453-463 (2014).

28. Heermann, T., Ramm, B., Glaser, S. \& Schwille, P. Local Self-Enhancement of MinD Membrane Binding in Min Protein Pattern Formation. Journal of Molecular Biology 432 , 3191-3204 (2020).

29. Heermann, T., Steiert, F., Ramm, B., Hundt, N. \& Schwille, P. Mass-sensitive particle tracking to elucidate the membrane-associated MinDE reaction cycle. Nat Methods 18, 1239-1246 (2021).

30. Würthner, L. et al. Bridging scales in a multiscale pattern-forming system. arXiv:2111.12043 [nlin, physics:physics] (2021).

31. Vecchiarelli, A. G. et al. Membrane-bound MinDE complex acts as a toggle switch that drives Min oscillation coupled to cytoplasmic depletion of MinD. Proc Natl Acad Sci USA 113, E1479-E1488 (2016).

32. Glock, P. \& Schwille, P. Switching protein patterns on membranes. Current Opinion in Colloid \& Interface Science 38, 100-107 (2018).

33. Zieske, K., Schweizer, J. \& Schwille, P. Surface topology assisted alignment of Min protein waves. FEBS Letters 588, 2545-2549 (2014).

34. Caspi, Y. \& Dekker, C. Mapping out Min protein patterns in fully confined fluidic chambers. eLife 5, e19271 (2016).

35. Ramm, B. et al. A diffusiophoretic mechanism for ATP-driven transport without motor proteins. Nat. Phys. 17, 850-858 (2021).

36. Glock, P. et al. Optical Control of a Biological Reaction-Diffusion System. Angew. Chem. 130, 2386-2390 (2018).

37. Litschel, T., Ramm, B., Maas, R., Heymann, M. \& Schwille, P. Beating Vesicles: Encapsulated Protein Oscillations Cause Dynamic Membrane Deformations. Angew. Chem. Int. Ed. 57, 16286-16290 (2018).

38. Godino, E. et al. De novo synthesized Min proteins drive oscillatory liposome deformation and regulate FtsA-FtsZ cytoskeletal patterns. Nat Commun 10, 4969 (2019).

39. Rovinsky, A. B. \& Menzinger, M. Chemical instability induced by a differential flow. Phys. Rev. Lett. 69, 1193-1196 (1992). 
40. Gholami, A., Steinbock, O., Zykov, V. \& Bodenschatz, E. Flow-driven instabilities during pattern formation of Dictyostelium discoideum. New J. Phys. 17, 063007 (2015).

41. Meindlhumer, S., Kerssemakers, J. \& Dekker, C. Practical Guide to Min pattern analysis. Unpublished. Article in preparation.

42. Wigbers, M. C., Brauns, F., Leung, C. Y. \& Frey, E. Flow Induced Symmetry Breaking in a Conceptual Polarity Model. Cells 9, 1524 (2020).

43. Goldstein, R. E., Tuval, I. \& van de Meent, J.-W. Microfluidics of cytoplasmic streaming and its implications for intracellular transport. Proceedings of the National Academy of Sciences 105, 3663-3667 (2008).

44. Hoijman, E., Rubbini, D., Colombelli, J. \& Alsina, B. Mitotic cell rounding and epithelial thinning regulate lumen growth and shape. Nat Commun 6, 7355 (2015).

45. Dasgupta, A. et al. Cell volume changes contribute to epithelial morphogenesis in zebrafish Kupffer's vesicle. eLife 7, e30963 (2018).

46. Goryachev, A. B. \& Leda, M. Many roads to symmetry breaking: molecular mechanisms and theoretical models of yeast cell polarity. MBoC 28, 370-380 (2017).

47. Goryachev, A. B. \& Leda, M. Compete or Coexist? Why the Same Mechanisms of Symmetry Breaking Can Yield Distinct Outcomes. Cells 9, 2011 (2020).

48. Bonny, M., Fischer-Friedrich, E., Loose, M., Schwille, P. \& Kruse, K. Membrane Binding of MinE Allows for a Comprehensive Description of Min-Protein Pattern Formation. PLoS Comput Biol 9, e1003347 (2013).

49. Loose, M., Fischer-Friedrich, E., Ries, J., Kruse, K. \& Schwille, P. Spatial Regulators for Bacterial Cell Division Self-Organize into Surface Waves in Vitro. Science 320, 789-792 (2008).

50. Bois, J. S., Jülicher, F. \& Grill, S. W. Pattern Formation in Active Fluids. Phys. Rev. Lett. 106, 028103 (2011).

51. Eckstein, T. et al. Influence of fast advective flows on pattern formation of Dictyostelium discoideum. PLoS ONE 13, e0194859 (2018).

52. Eckstein, T., Vidal-Henriquez, E. \& Gholami, A. Experimental observation of boundarydriven oscillations in a reaction-diffusion-advection system. Soft Matter 16, 4243-4255 (2020). 


\section{Figures}

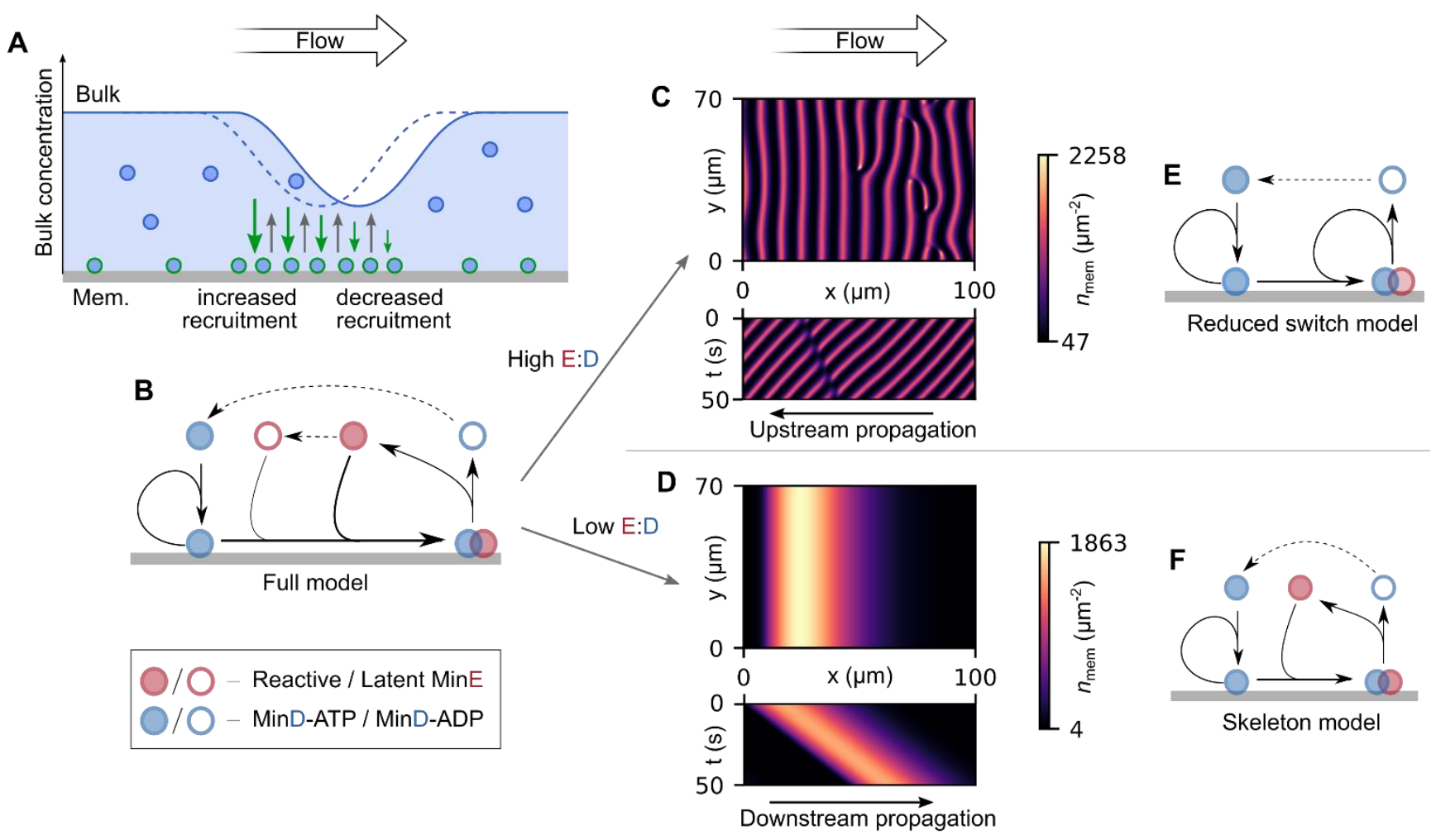

Fig. 1: Min models and simulation results.

A Illustration of the effect that bulk flow has on pattern formation. MinD advection in the bulk shifts its concentration profile in the bulk relative to the membrane pattern, leading to an increase in the bulk concentration on the upstream side of wave crests relative to the downstream side. This enhances the recruitment rate (green arrows) on the upstream side relative to the downstream side and thus, results in a movement of the membrane pattern (but not the individual proteins) in the upstream direction. $\mathbf{B}$ Diagram depicting the interactions in the full switch model. This model includes the MinE switch. MinDATP binds the membrane, recruiting more MinD-ATP as well as reactive MinE. After MinE stimulates ATP-hydrolysis, MinD-ADP and MinE detach from the membrane. MinD needs to ADP for ATP in the bulk. MinE temporarily assumes a latent state before rebinding to the membrane. C Typical profile shape (snapshots) and kymograph of the membrane protein density at high E:D ratios. D Typical profile shape (snapshots) and kymograph of the membrane protein density at low E:D ratio. E For high E:D ratios the full switch model can be simplified into the reduced switch model. MinE bulk gradients become negligible. $\mathbf{F}$ At low $\mathrm{E}: \mathrm{D}$ ratios, the behaviour of the Min system is captured well by the skeleton model. This model does not include the MinE switch. 
bioRxiv preprint doi: https://doi.org/10.1101/2021.12 23.474007; this version posted December 23, 2021. The copyright holder for this preprint (which was not certified by peer review) is the author/funder, who has granted bioRxiv a license to display the preprint in perpetuity. It is made available under aCC-BY-NC-ND 4.0 International license.

MinD-Cy3 in magenta MinE-Cy5 in green

A

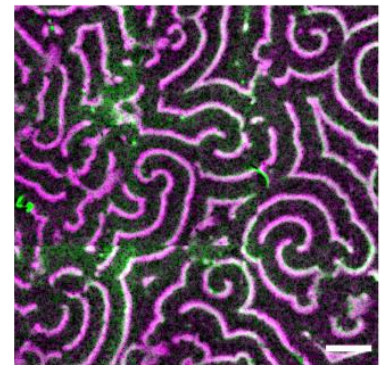

B

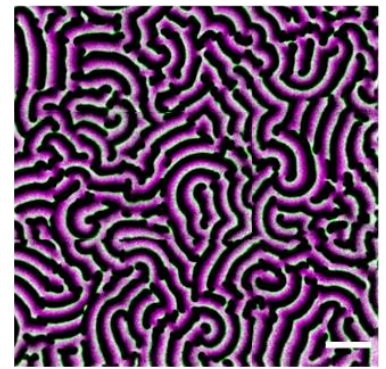

C

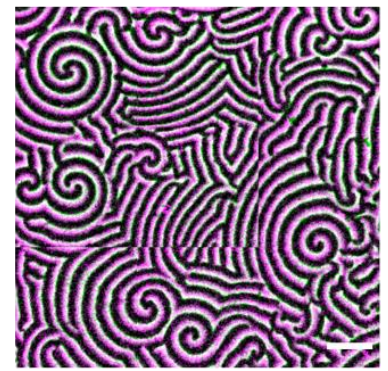

no flow

flow
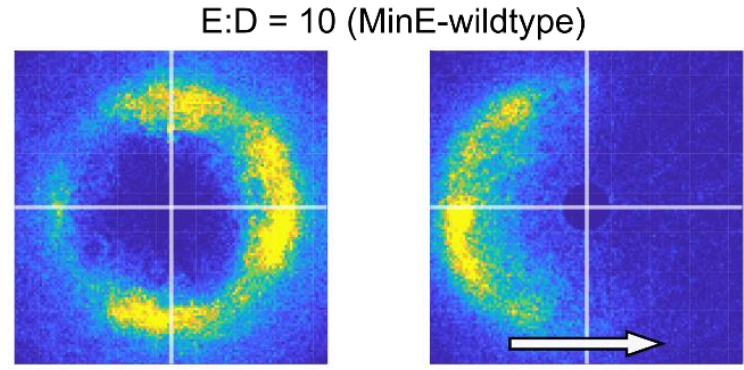

$0.11 \mathrm{~mm} / \mathrm{s}$

$E: D=2$ (MinE-wildtype)
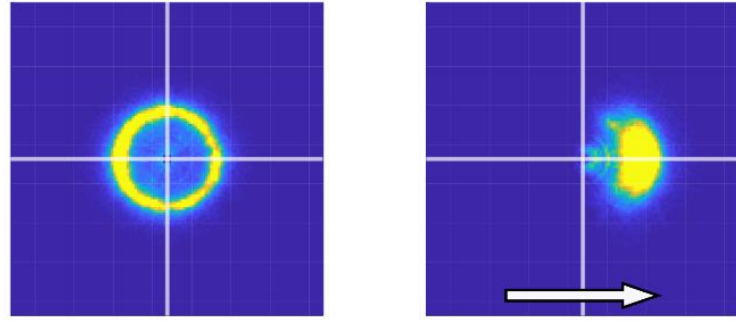

$0.21 \mathrm{~mm} / \mathrm{s}$

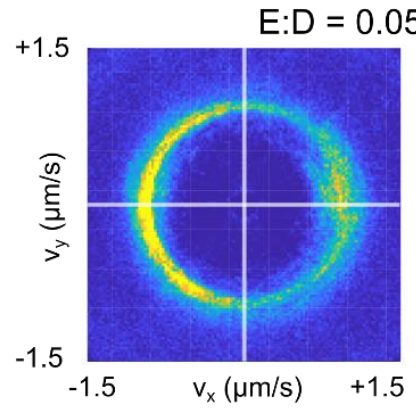

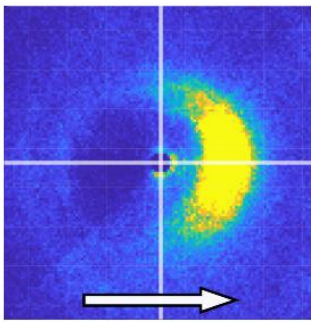

$0.15 \mathrm{~mm} / \mathrm{s}$
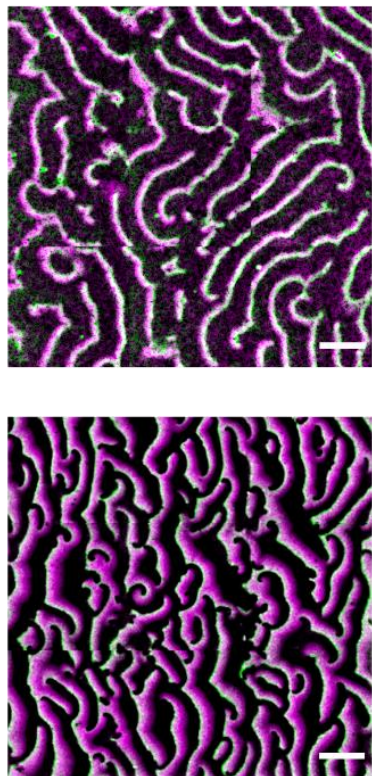

$\max$

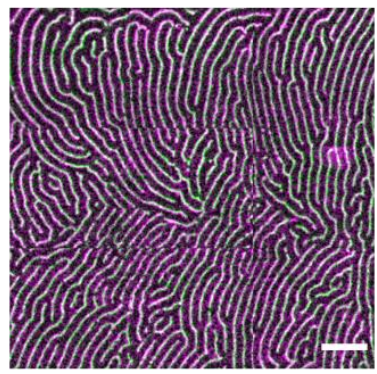

Fig. 2: Experimental data showing how patterns respond to flow at different E:D ratios.

A Upstream propagation was observed for high E:D ratio (initial 10). B Downstream propagation observed for low E:D ratio (initial 2, corrected 1.3). C Downstream propagation observed for MinE$\mathrm{L} 3 \mathrm{I} 24 \mathrm{~N}$ at $\mathrm{E}: \mathrm{D}=0.05$

Data are for MinE-wildtype (A, B) and MinE-L3I24N (C), with bulk flow directed left-to-right. Min patterns (outer left and outer right columns) show MinD-Cy3 in magenta and MinE-Cy5 in green. All scale bars are $100 \mu \mathrm{m}$. The results of wave-propagation analysis are represented as $2 \mathrm{D}$ histograms (center columns), showing counts for directionality $\left(v_{x}, v_{y}\right)$. Left half of the figures displays an exemplary image as well as wave-propagation analysis for the no-flow case. Right half of the figures displays an exemplary image as well as wave-propagation analysis with flow. Images stitched from 3x3 images. 


\section{A}

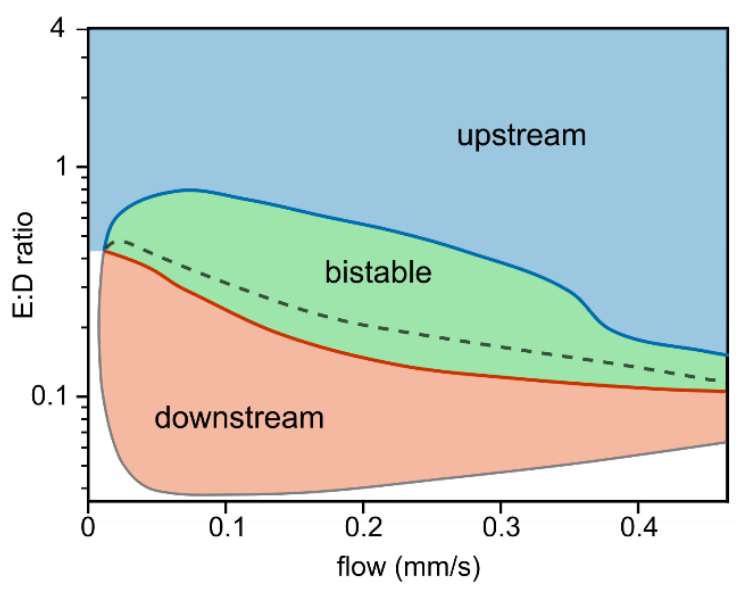

B

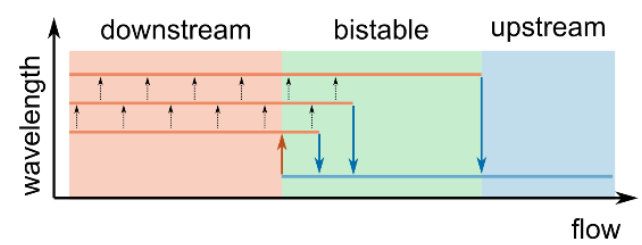

Fig. 3: Phase diagram displaying the predicted direction of pattern propagation.

A Phase diagram displaying the predicted direction of pattern propagation. Red and blue regions indicate the parts of the parameter space where exclusively downstream or upstream patterns are observed, respectively. Green region indicates the bistability regime, where the propagation direction depends on the initial conditions. If simulations are initiated from the homogeneous steady state, the observed propagation direction is downstream below the black dashed line, and upstream above it. B Schematic visualizing that the transition flow velocity depends on the wavelength of the pattern. Upon increasing the flow velocity, larger wavelength patterns reverse the propagation direction at a higher flow velocity. 
A

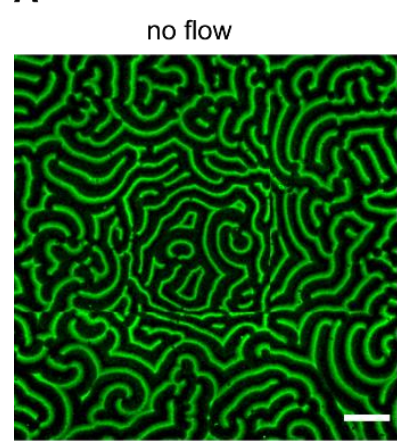

B
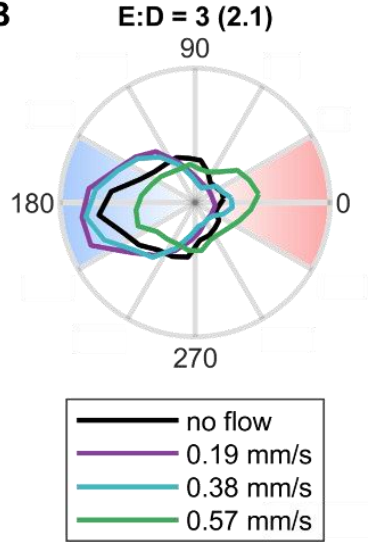

$\mathbf{F}$

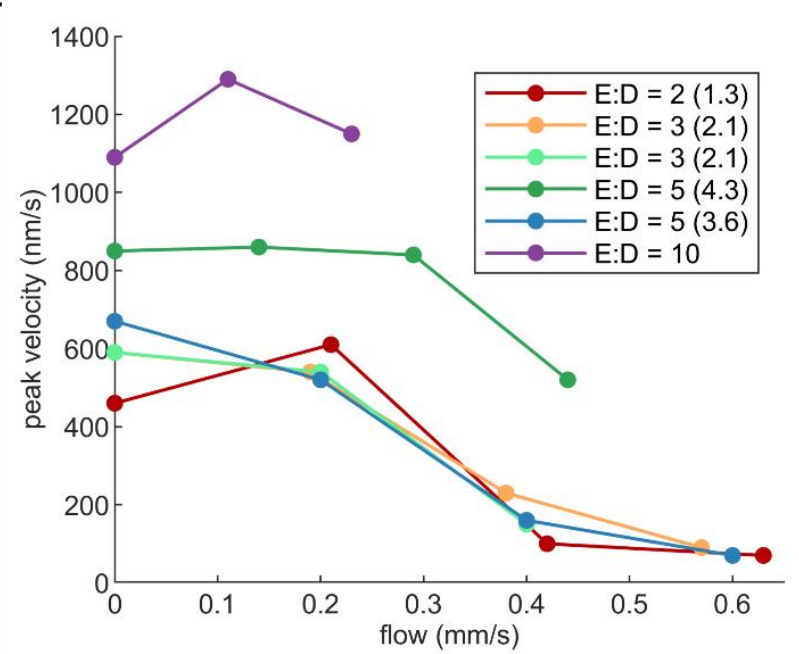

$E: D=5(3.6)$

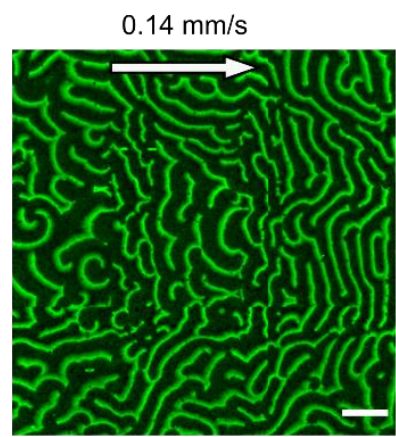

C
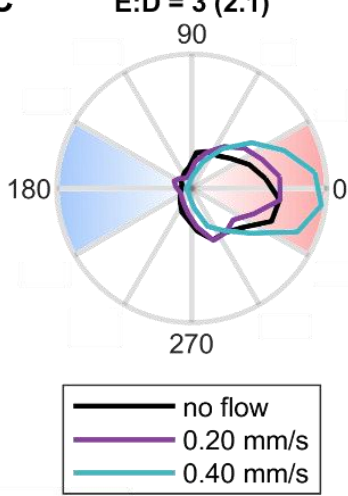

/s

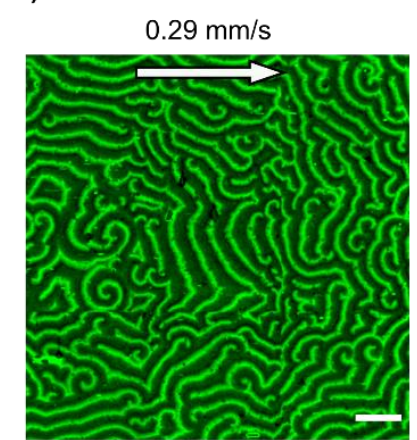

D
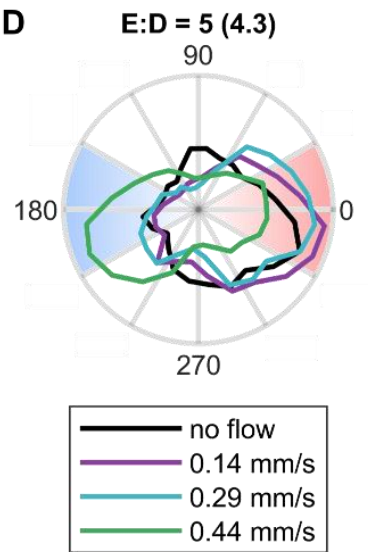

MinE-Cy5 in green

$0.44 \mathrm{~mm} / \mathrm{s}$

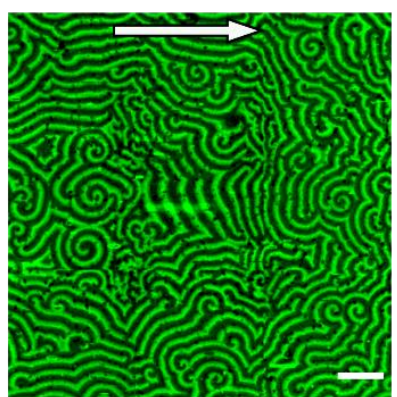

E $\quad E: D=5(3.6)$

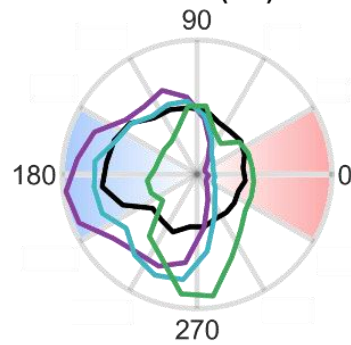

270

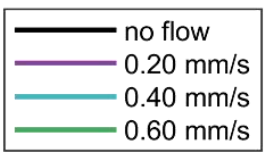

G

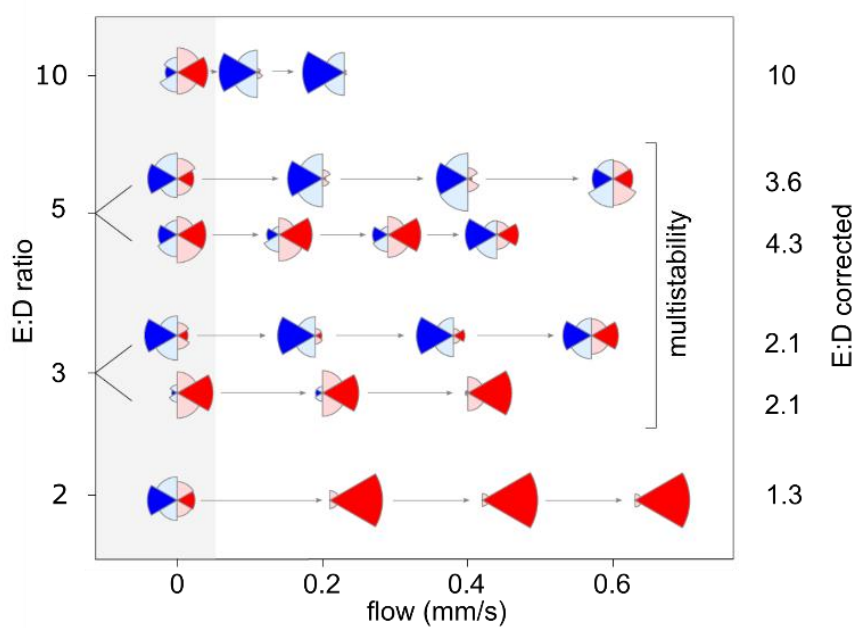

Fig. 4: Experimental data showing how Min patterns respond to a sequence of flow rates.

A Min patterns for different flow velocities at initial ratio $E: D=5$ (corrected 3.6). Channel MinE-Cy5 in green, scale bars $100 \mu \mathrm{m}$. Images stitched from 3x3 grids. B Polar histogram showing counts per angular segment for initial ratio $E: D=5$ (corrected 4.3). C Idem for initial ratio $E: D=5$ (corrected 3.6). D Idem for initial ratio $E: D=3$ (corrected 2.1). E Idem for initial ratio $E: D=3$ (corrected 2.1). F Peak velocity (obtained maximum of histogram in bins of $10 \mathrm{~nm} / \mathrm{s}$ ) obtained from MinE-Cy5 channel, shown as function of flow rate for different E:D ratios. G Overview of experiments done for wildtype MinE. Downstream and upstream fractions are obtained from histogram counts per angular segment. Size of 
bioRxiv preprint doi: https://doi.org/10.1101/2021.12.23.474007; this version posted December 23, 2021. The copyright holder for this preprint (which was not certified by peer review) is the author/funder, who has granted bioRxiv a license to display the preprint in perpetuity. It is made available under aCC-BY-NC-ND 4.0 International license.

fraction is represented in the segment's radius. Red represents downstream flowing patterns, blue represents upstream patterns. 